\title{
THE CORRELATION FUNCTIONS OF THE $X X Z$ HEISENBERG CHAIN IN THE CASE OF ZERO OR INFINITE ANISOTROPY, AND RANDOM WALKS OF VICIOUS WALKERS
}

\author{
N. M. BOGOLIUBOV AND C. MALYSHEV
}

Dedicated to L. D. Faddeev

\begin{abstract}
The $X X Z$ Heisenberg chain is considered for two specific limits of the anisotropy parameter: $\Delta \rightarrow 0$ and $\Delta \rightarrow-\infty$. The corresponding wave functions are expressed in terms of symmetric Schur functions. Certain expectation values and thermal correlation functions of the ferromagnetic string operators are calculated over the basis of $N$-particle Bethe states. The thermal correlator of the ferromagnetic string is expressed through the generating function of the lattice paths of random walks of vicious walkers. A relationship between the expectation values obtained and the generating functions of strict plane partitions in a box is discussed. An asymptotic estimate of the thermal correlator of the ferromagnetic string is obtained in the zero temperature limit. It is shown that its amplitude is related to the number of plane partitions.
\end{abstract}

\section{§1. INTRODUCTION}

1.1. $X X Z$ Heisenberg chain. A system of spin $1 / 2$ particles occupying sites of a one-dimensional lattice, widely known as the quantum $X Y Z$ Heisenberg chain 1, has attracted considerable attention both in theoretical and mathematical physics, and it has been thoroughly investigated for a long time [2] $[$ ]. The quantum inverse scattering method, developed for solving integrable models of quantum field theory and statistical physics [9, 10, has also been employed to investigate the $X Y Z$ Heisenberg chain [11, 12]. An important special case of the $X Y Z$ model, the so-called $X X Z$ spin chain, also attracts considerable attention [13, 14, [18]-20. The Hamiltonian of the $X X Z$ magnet was diagonalized by the coordinate Bethe Ansatz method in [3]-5]. The algebraic Bethe Ansatz was used in 11 to solve the $X X Z$ model. The calculation of correlation functions of the model in question in the framework of the algebraic Bethe Ansatz has required serious efforts [13] -17 .

The random walks is a classical problem both for combinatorics and statistical physics. The problem of enumeration of the paths made by the so-called vicious walkers on the one-dimensional lattice was formulated and investigated in detail by Fisher [21. This problem still continues to attract considerable attention of physicists and mathematicians [22]-34].

2010 Mathematics Subject Classification. Primary 81U40.

Key words and phrases. XXZ Heisenberg chain, Schur functions, random walks, plane partitions.

Extended talk at the Conference "Conformal Field Theory, Integrable Systems, and Liouville Gravity" (Chernogolovka, June 30-July 2, 2009).

Partially supported by RFBR (No. 07-01-00358) and by the Russian Academy of Sciences program "Mathematical Methods in Nonlinear Dynamics". 
Random walks on a one-dimensional periodic lattice can be related to the correlation functions of the $X X$ Heisenberg magnet. Certain operator averages taken over the ferromagnetic state of the $X X$ model play a role of the generating functions of the number of paths traced by vicious walkers. The enumeration problem for trajectories of vicious walkers in terms of correlation functions of the $X X$ model has been studied in the series of papers [35]-38]. The approach of 35]-38] will be explored further in the present paper. We shall consider two limits of the $X X Z$ spin chain from the viewpoint of random walks of vicious walkers, as well as from the viewpoint of enumeration of boxed plane partitions.

We start with the $X X Z$ model defined on the one-dimensional lattice consisting of $M+1$ sites labeled by elements of the set $\mathcal{M} \equiv\{0 \leq k \leq M, k \in \mathbb{Z}\}, M+1=0(\bmod 2)$. In the absence of an external magnetic field, the corresponding spin Hamiltonian is defined as follows:

$$
\widehat{H}_{X X Z}=-\frac{1}{2} \sum_{k=0}^{M}\left(\sigma_{k+1}^{-} \sigma_{k}^{+}+\sigma_{k+1}^{+} \sigma_{k}^{-}+\frac{\Delta}{2}\left(\sigma_{k+1}^{z} \sigma_{k}^{z}-1\right)\right),
$$

where the parameter $\Delta \in \mathbb{R}$ describes the internal anisotropy of the model. For instance, the choice $\Delta= \pm 1$ corresponds to the so-called isotropic $X X X$ spin chain solved in [2]. The local spin operators $\sigma_{k}^{ \pm}=\frac{1}{2}\left(\sigma_{k}^{x} \pm i \sigma_{k}^{y}\right)$ and $\sigma_{k}^{z}$, depending on the lattice argument $k \in \mathcal{M}$, are defined as $(M+1)$-fold tensor products as follows:

$$
\sigma_{k}^{\#}=\sigma^{0} \otimes \cdots \otimes \sigma^{0} \otimes \underbrace{\sigma^{\#}}_{k} \otimes \sigma^{0} \otimes \cdots \otimes \sigma^{0},
$$

where $\sigma^{0}$ is the $2 \times 2$ unit matrix, and $\sigma^{\#}$ at the $k$ th place denotes a Pauli matrix, $\sigma^{\#} \in \mathfrak{s u}(2)$ (the superscript \# implies either $x, y, z$ or \pm ). Therefore, the spin operators act over the state-space $\mathfrak{H}_{M+1}$ given by the tensor product of $M+1$ copies of the linear spaces $\mathfrak{h}_{k} \equiv \mathbb{C}^{2}: \mathfrak{H}_{M+1}=\bigotimes_{k=0}^{M} \mathfrak{h}_{k}$. The commutation rules for the spin operators are given by the relations

$$
\left[\sigma_{k}^{+}, \sigma_{l}^{-}\right]=\delta_{k, l} \sigma_{l}^{z}, \quad\left[\sigma_{k}^{z}, \sigma_{l}^{ \pm}\right]= \pm 2 \delta_{k, l} \sigma_{l}^{ \pm} .
$$

The linear space $\mathbb{C}^{2}$ is spanned over the spin "up" and "down" states $(|\uparrow\rangle$ and $|\downarrow\rangle$, respectively), providing a natural basis so that

$$
|\uparrow\rangle \equiv\left(\begin{array}{l}
1 \\
0
\end{array}\right), \quad|\downarrow\rangle \equiv\left(\begin{array}{l}
0 \\
1
\end{array}\right) .
$$

The space $\mathfrak{H}_{M+1}$ is spanned over the state vectors $\bigotimes_{k=0}^{M}|s\rangle_{k}$, where $s$ means either $\uparrow$ or $\downarrow$. The periodic boundary conditions $\sigma_{k+(M+1)}^{\#}=\sigma_{k}^{\#}$ are assumed.

To represent $N$-particle state vectors $\left|\Psi_{N}\left(u_{1}, \ldots, u_{N}\right)\right\rangle$ of the model, let the sites with spin "down" states be labeled by the coordinates $\mu_{i}, 1 \leq i \leq N$. These coordinates form a strict partition $\boldsymbol{\mu} \equiv\left(\mu_{1}, \mu_{2}, \ldots, \mu_{N}\right)$, where $M \geq \mu_{1}>\mu_{2}>\cdots>\mu_{N} \geq 0$. There is a correspondence between each partition and the corresponding sequence of zeros and units of the form: $\left\{e_{k} \equiv e_{k}(\boldsymbol{\mu})\right\}_{k \in \mathcal{M}}$, where $e_{k} \equiv \delta_{k, \mu_{n}}, 1 \leq n \leq N$. This correspondence enables another convenient expression for the strict partitions: $\boldsymbol{\mu}=\left(M^{e_{M}}, \ldots, 1^{e_{1}}, 0^{e_{0}}\right)$. Here it is meant that any nonnegative

integer $k \in \mathcal{M}$ appears $e_{k}$ times in the present configuration, and the condition $\sum_{k=0}^{M} e_{k}=N$ is respected. The Hamiltonian (1) is diagonalized via the following Ansatz:

$$
\left|\Psi_{N}(\mathbf{u})\right\rangle=\sum_{\left\{e_{k}(\boldsymbol{\mu})\right\}_{k \in \mathcal{M}}} \chi_{\boldsymbol{\mu}}^{X X Z}(\mathbf{u}) \prod_{k=0}^{M}\left(\sigma_{k}^{-}\right)^{e_{k}}|\Uparrow\rangle,
$$


where the sum is taken over $C_{M}^{N}$ strict partitions $\boldsymbol{\mu}$. The state $|\Uparrow\rangle$ in (3) is the fully polarized state with all spins "up": $|\uparrow\rangle \equiv \bigotimes_{n=0}^{M}|\uparrow\rangle_{n}$. Moreover, it is proposed to use bold-faced letters as short-hand notation for appropriate $N$-tuples of numbers: for instance, $\mathbf{u}$ instead of $\left(u_{1}, \ldots, u_{N}\right)$, etc. Therefore, the wave function $\chi_{\boldsymbol{\mu}}^{X X Z}(\mathbf{u})$ in (3) takes the form

$$
\chi_{\boldsymbol{\mu}}^{X X Z}(\mathbf{u})=\sum_{S_{p_{1}, p_{2}, \ldots, p_{N}}} \mathcal{A}_{S}(\mathbf{u}) u_{p_{1}}^{2 \mu_{1}} u_{p_{2}}^{2 \mu_{2}} \cdots u_{p_{N}}^{2 \mu_{N}},
$$

where summation over all elements of the symmetric group of permutations $S_{p_{1}, p_{2}, \ldots, p_{N}}$ $\equiv S\left(\begin{array}{cccc}1, & 2, & \ldots, & N \\ p_{1}, & p_{2}, & \ldots, & p_{N}\end{array}\right)$. The amplitude $\mathcal{A}_{S}$ is given by the product

$$
\mathcal{A}_{S}(\mathbf{u}) \equiv \prod_{1 \leq j<i \leq N} \frac{1-2 \Delta u_{p_{i}}^{2}+u_{p_{i}}^{2} u_{p_{j}}^{2}}{u_{p_{i}}^{2}-u_{p_{j}}^{2}} .
$$

The state vectors (3) are eigenstates of the Hamiltonian (11),

$$
\widehat{H}_{X X Z}\left|\Psi_{N}(\mathbf{u})\right\rangle=E_{N}(\mathbf{u})\left|\Psi_{N}(\mathbf{u})\right\rangle
$$

if and only if the variables $u_{l}(1 \leq l \leq N)$ satisfy the Bethe equations

$$
u_{l}^{2(M+1)}=(-1)^{N-1} \prod_{k=1}^{N} \frac{1-2 \Delta u_{l}^{2}+u_{l}^{2} u_{k}^{2}}{1-2 \Delta u_{k}^{2}+u_{l}^{2} u_{k}^{2}} .
$$

The corresponding eigenenergies are given by

$$
E_{N}(\mathbf{u})=-\frac{1}{2} \sum_{i=1}^{N}\left(u_{i}^{2}+u_{i}^{-2}-2 \Delta\right) .
$$

1.2. Outline of the limiting models and setting of the problem. In our paper we shall consider two special cases of the $X X Z$ model, namely the $\Delta \rightarrow 0$ and $\Delta \rightarrow-\infty$ limits. The $\Delta \rightarrow 0$ limit, known as the $X X$ Heisenberg chain, is most popular and most studied [6], 40]-46]. The Hamiltonian of the $X X$ model describes the nearest-neighbor interactions of spin "up" $|\uparrow\rangle$ and spin "down" $|\downarrow\rangle$ states located on sites of the periodic chain in a zero magnetic field; it has the form

$$
\widehat{H}_{X X} \equiv-\frac{1}{2} \sum_{k=0}^{M}\left(\sigma_{k+1}^{-} \sigma_{k}^{+}+\sigma_{k+1}^{+} \sigma_{k}^{-}\right) .
$$

It is crucial that the system described by the Hamiltonian (8) is equivalent to free fermions 41. The case of the $X X$ magnet can also be deduced by taking the limit of infinite onsite repulsion in the boson Hubbard model (the "hard-core" bosons) [4]. Therefore, the $X X$ model is of interest for investigating the (quantum) phase diagram of the Hubbard model, as well as for description of the Frenkel excitons [48. In the past few years, the $X X$ model has attracted attention in connection with quantum information theory and computation theory [49, 50].

The state vector of the $X X$ model and the corresponding Bethe equations are obtained at $\Delta=0$ from (4) and (6), respectively. Up to an irrelevant prefactor, the wave functions of the model are equal to

$$
\chi_{\boldsymbol{\mu}}^{X X}(\mathbf{u})=\operatorname{det}\left(u_{j}^{2 \mu_{k}}\right)_{1 \leq j, k \leq N} \prod_{1 \leq n<l \leq N}\left(u_{l}^{2}-u_{n}^{2}\right)^{-1},
$$

and the Bethe equations (see [41]) look like the following:

$$
u_{j}^{2(M+1)}=(-1)^{N-1}, \quad 1 \leq j \leq N .
$$


The substitution $u_{j}^{2}=e^{i \theta_{j}}$ brings these equations to the exponential form,

$$
e^{i(M+1) \theta_{j}}=(-1)^{N-1},
$$

with the solutions

$$
\theta_{j}=\frac{2 \pi}{M+1}\left(I_{j}-\frac{N-1}{2}\right),
$$

where the $I_{j}$ are integers or half-integers, depending on whether $N$ is odd or even. It suffices to consider a set of $N$ different numbers $I_{j}$ satisfying $M \geq I_{1}>I_{2}>\cdots>I_{N} \geq 0$. The notation $\boldsymbol{\theta}$ for the $N$-tuple $\left(\theta_{1}, \theta_{2}, \ldots, \theta_{N}\right)$ of the solutions (12) will be especially convenient below in order to stress that we are concerned with the solution of the Bethe equation. Otherwise, it is appropriate to use $\mathbf{u}$ as an indication that an arbitrary set of parameters is meant. From (77) it follows that the eigenenergies of the $X X$ model are equal to

$$
E_{N}^{X X}(\boldsymbol{\theta})=-\sum_{j=1}^{N} \cos \theta_{j}=-\sum_{j=1}^{N} \cos \left(\frac{2 \pi}{M+1}\left(I_{j}-\frac{N-1}{2}\right)\right) .
$$

The ground state of the model corresponds to the following solutions of the Bethe equations:

$$
\theta_{j}=\frac{2 \pi}{M+1}\left(N-j-\frac{N-1}{2}\right), \quad 1 \leq j \leq N .
$$

The strong anisotropy (SA) limit as $\Delta \rightarrow-\infty$ for the $X X Z$ model is less studied [4, 8, 51- 54. In this limit, the behavior of the system is described by the effective Hamiltonian that is formally equivalent to the $X X$ Hamiltonian supplied with a requirement forbidding two spin "down" states to occupy any pair of nearest-neighboring sites [51, 52]:

$$
\widehat{H}_{\mathrm{SA}}=-\frac{1}{2} \sum_{k=0}^{M} \mathcal{P}\left(\sigma_{k+1}^{-} \sigma_{k}^{+}+\sigma_{k+1}^{+} \sigma_{k}^{-}\right) \mathcal{P},
$$

where the projector $\mathcal{P}$ cuts out the states with the spin "down" at any pair of nearestneighboring sites: $\mathcal{P} \equiv \prod_{k=0}^{M}\left(1-\widehat{q}_{k+1} \widehat{q}_{k}\right)$. The local projectors onto the spin "up" and "down" states are of the form

$$
\breve{q}_{k} \equiv \frac{1}{2}\left(\sigma_{k}^{0}+\sigma_{k}^{z}\right), \quad \widehat{q}_{k} \equiv \frac{1}{2}\left(\sigma_{k}^{0}-\sigma_{k}^{z}\right), \quad \check{q}_{k}+\widehat{q}_{k}=\mathbb{I}, \quad k \in \mathcal{M},
$$

where the operators $\sigma_{k}^{\#}$ are defined by (2).

In the limit $\Delta \rightarrow-\infty$, the wave function (4) takes the form

$$
\chi_{\boldsymbol{\mu}}^{\mathrm{SA}}(\mathbf{u})=\operatorname{det}\left(u_{j}^{2\left(\mu_{k}-N+k\right)}\right)_{1 \leq j, k \leq N} \prod_{1 \leq n<l \leq N}\left(u_{l}^{2}-u_{n}^{2}\right)^{-1},
$$

where the coordinates of the spin "down" states form a strict decreasing partition $\boldsymbol{\mu}$ (as in (41) and (91)), i.e., $M \geq \mu_{1}>\mu_{2}>\cdots>\mu_{N} \geq 0$. Formula (17) shows that the wave function is not equal to zero if and only if the elements $\mu_{i}, 1 \leq i \leq N$, satisfy the exclusion condition: $\mu_{i}>\mu_{i+1}+1$. It is crucial that in the limit in question, the occupation of nearest sites is forbidden, and the hard-core diameter, equal to the duplicated intersite separation, arises. The Bethe equations of the model take the form

$$
e^{i(M+1-N) \theta_{k}}=(-1)^{N-1} \prod_{j=1}^{N} e^{-i \theta_{j}}, \quad 1 \leq k \leq N,
$$


and have the solutions

$$
\theta_{j}=\frac{2 \pi}{M+1-N}\left(I_{j}-\frac{N-1}{2}-P\right),
$$

where $P \equiv \frac{1}{2 \pi} \sum_{j=1}^{N} \theta_{j}$, and the $I_{j}$ are integers or half-integers, depending on $N$ being odd or even, and satisfy the condition $M-N \geq I_{1}>I_{2}>\cdots>I_{N} \geq 0$. The ground state of the model is defined by the solutions

$$
\theta_{j}=\frac{2 \pi}{M+1-N}\left(N-j-\frac{N-1}{2}\right)
$$

with $P=0$. The eigenenergy of the model is

$$
E_{N}(\boldsymbol{\theta})=-\sum_{j=1}^{N} \cos \left(\frac{2 \pi}{M+1-N}\left(I_{j}-\frac{N-1}{2}-P\right)\right) .
$$

The two limits (8) and (15) are similar in that their wave functions are expressible through the Schur functions [55]:

$$
\begin{aligned}
S_{\boldsymbol{\lambda}}(\mathbf{x}) & \equiv S_{\boldsymbol{\lambda}}\left(x_{1}, x_{2}, \ldots, x_{N}\right) \equiv \frac{\operatorname{det}\left(x_{j}^{\lambda_{k}+N-k}\right)_{1 \leq j, k \leq N}}{\operatorname{det}\left(x_{j}^{N-k}\right)_{1 \leq j, k \leq N}} \\
& =\operatorname{det}\left(x_{j}^{\lambda_{k}+N-k}\right)_{1 \leq j, k \leq N} \prod_{1 \leq n<l \leq N}\left(x_{l}-x_{n}\right)^{-1},
\end{aligned}
$$

where $\boldsymbol{\lambda}$ denotes a partition $\left(\lambda_{1}, \lambda_{2}, \ldots, \lambda_{N}\right)$, which is an $N$-tuple of nonincreasing nonnegative integers, $L \geq \lambda_{1} \geq \lambda_{2} \geq \cdots \geq \lambda_{N} \geq 0$. Indeed, any strict partition $M \geq \mu_{1}>\mu_{2}>\cdots>\mu_{N} \geq 0$ and a nonstrict partition $M+1-N \geq \lambda_{1} \geq$ $\lambda_{2} \geq \cdots \geq \lambda_{N} \geq 0$ (denoted as $\boldsymbol{\mu}$ and $\boldsymbol{\lambda}$, respectively) can be related by the formula $\lambda_{j}=\mu_{j}-N+j$, where $1 \leq j \leq N$. In other terms, $\boldsymbol{\lambda}=\boldsymbol{\mu}-\boldsymbol{\delta}$, where $\boldsymbol{\delta}$ is the strict partition $(N-1, N-2, \ldots, 1,0)$. So, the wave function of the $X X$ model (9) can be represented as

$$
\chi_{\boldsymbol{\mu}}^{X X}(\mathbf{u})=S_{\boldsymbol{\lambda}}\left(\mathbf{u}^{2}\right)
$$

Any strict partition $\boldsymbol{\mu}$ with elements respecting the exclusion condition $\mu_{i}>\mu_{i+1}+1$ is connected with a nonstrict partition $\widetilde{\boldsymbol{\lambda}}$ by the relation $\widetilde{\boldsymbol{\lambda}}=\boldsymbol{\mu}-2 \boldsymbol{\delta}$, where $M+2(1-N) \geq$ $\widetilde{\lambda}_{1} \geq \widetilde{\lambda}_{2} \geq \cdots \geq \widetilde{\lambda}_{N} \geq 0$. Therefore, equation (17) takes the form

$$
\chi_{\boldsymbol{\mu}}^{\mathrm{SA}}(\mathbf{u})=S_{\widetilde{\boldsymbol{\lambda}}}\left(\mathbf{u}^{2}\right) .
$$

It is useful to recall a graphical picture (see Figure 1) of the correspondence between the strict partitions $\boldsymbol{\mu}$ and the nonstrict partitions $\boldsymbol{\lambda}$ used in (23). Namely, with each partition $\boldsymbol{\lambda}$ we associate a set of $N$-tuples $\mathfrak{G}(\boldsymbol{\lambda})$ as follows [59]:

$$
\mathfrak{G}(\boldsymbol{\lambda})=\left\{\lambda_{j}-j+\frac{1}{2} \mid 1 \leq j \leq N\right\} \subset \mathbb{Z}+\frac{1}{2} .
$$

On the other hand, any nonstrict partition $\boldsymbol{\lambda}$ can be represented as a rectangular table (the Young table) consisting of $N$ columns so that, for all $i, \lambda_{i}$ is the height of the $i$ th column $\left(\lambda_{i} \leq M-N+1\right)$. We shift each element of the set $\mathfrak{G}(\boldsymbol{\lambda})$ by $N+\frac{1}{2}$. Then we assign the resulting numbers to the projections along the vertical dashed lines onto the horizontal axis. The set of points on the horizontal axis is precisely the strict partition $\boldsymbol{\mu}$. For instance, the diagram in Figure 1 is drawn for $M=8$ and $N=4$, and we have $\boldsymbol{\lambda}=(5,3,2,2)$ and $\boldsymbol{\mu}=(8,5,3,2)$. 


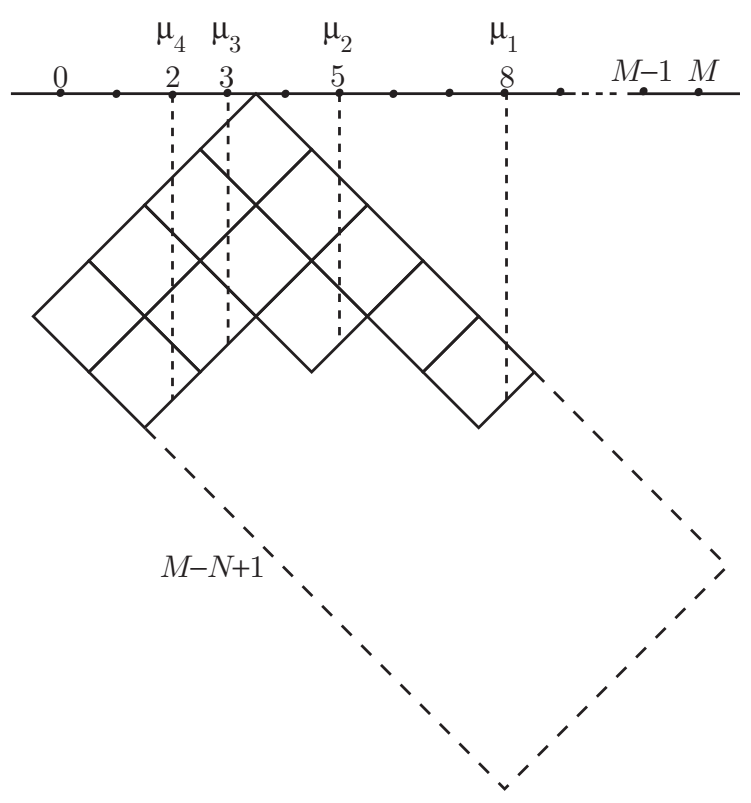

FiguRE 1. The strict partition $\left(\mu_{1}, \mu_{2}, \mu_{3}, \mu_{4}\right)$ and the corresponding Young table.

In our paper we shall study the thermal correlation function of the states (to be called ferromagnetic strings) with no spins down on the last $n+1$ sites of the lattice. We are going to consider the expectation value defined by the ratio

$$
\mathcal{T}(\boldsymbol{\theta}, n, \beta) \equiv \frac{\left\langle\Psi_{N}(\boldsymbol{\theta})\left|\bar{\Pi}_{n} e^{-\beta \widehat{H}} \bar{\Pi}_{n}\right| \Psi_{N}(\boldsymbol{\theta})\right\rangle}{\left\langle\Psi_{N}(\boldsymbol{\theta}) \mid \Psi_{N}(\boldsymbol{\theta})\right\rangle}, \quad \bar{\Pi}_{n} \equiv \prod_{j=M-n}^{M} \check{q}_{j},
$$

where $\beta \in \mathbb{C}$, and the projector $\bar{\Pi}_{n}$ is expressed in terms of $\breve{q}_{j}$ (16). Also, $\hat{H}$ in (25) denotes either $\hat{H}_{X X}$ (see (8) ), or $\hat{H}_{\mathrm{SA}}$ (see (15)), and $\boldsymbol{\theta}$ indicates that the eigenstate $\left|\Psi_{N}(\boldsymbol{\theta})\right\rangle$ is calculated for the solution of the Bethe equation (11) or (18), respectively. Our calculations will be based on the similarity of the problem under consideration and the enumeration problem for admissible lattice paths made by random vicious walkers. We shall extensively use the following relation for the Schur functions (22), which is due to the Binet-Cauchy formula [56]:

$$
\begin{aligned}
& \sum_{\boldsymbol{\lambda} \subseteq\left\{L^{N}\right\}} S_{\boldsymbol{\lambda}}\left(x_{1}^{2}, \ldots, x_{N}^{2}\right) S_{\boldsymbol{\lambda}}\left(y_{1}^{2}, \ldots, y_{N}^{2}\right) \\
& \quad=\operatorname{det}\left(T_{j k}\right)_{1 \leq j, k \leq N} \prod_{1 \leq k<j \leq N}\left(y_{j}^{2}-y_{k}^{2}\right)^{-1} \prod_{1 \leq m<l \leq N}\left(x_{l}^{2}-x_{m}^{2}\right)^{-1} .
\end{aligned}
$$

The entries of the matrix $T_{j k}$ are of the form

$$
T_{j k}=\frac{1-\left(x_{k} y_{j}\right)^{2(N+L)}}{1-\left(x_{k} y_{j}\right)^{2}} .
$$

Summation in (26) goes over all nonstrict partitions $\lambda$ into at most $N$ parts each of which does not exceed $L: L \geq \lambda_{1} \geq \lambda_{2} \geq \cdots \geq \lambda_{N} \geq 0$. The notation for the range of summation in (26) will be extensively used in the rest of the paper. 


\section{§2. XX Heisenberg Chain}

2.1. The Bethe states and form factors. Before calculating $\mathcal{T}(\boldsymbol{\theta}, n, \beta)$ (see (25)), we apply our approach to more familiar examples. First, we specialize, with regard to (23), the state vector (3) and its conjugate as follows:

$$
\begin{aligned}
\left|\Psi_{N}(\mathbf{u})\right\rangle= & \sum_{\boldsymbol{\lambda} \subseteq\left\{(M+1-N)^{N}\right\}} S_{\boldsymbol{\lambda}}\left(\mathbf{u}^{2}\right) \prod_{k=0}^{M}\left(\sigma_{k}^{-}\right)^{e_{k}}|\Uparrow\rangle, \\
\left\langle\Psi_{N}(\mathbf{v})\right|= & \sum_{\boldsymbol{\lambda} \subseteq\left\{(M+1-N)^{N}\right\}}\langle\Uparrow| \prod_{k=0}^{M}\left(\sigma_{k}^{+}\right)^{\widetilde{e}_{k}} S_{\boldsymbol{\lambda}}\left(\mathbf{v}^{-2}\right),
\end{aligned}
$$

where the summation is over all nonstrict partitions $\boldsymbol{\lambda}$ related to the nonstrict partitions $\boldsymbol{\mu}=\boldsymbol{\lambda}+\boldsymbol{\delta}$. Because of the orthogonality relation

$$
\left\langle\Uparrow\left|\prod_{k=0}^{M}\left(\sigma_{k}^{+}\right)^{\widetilde{e}_{k}} \prod_{l=0}^{M}\left(\sigma_{l}^{-}\right)^{e_{l}}\right| \Uparrow\right\rangle=\prod_{n=0}^{M} \delta_{\widetilde{e}_{n} e_{n}}
$$

the scalar product of the state vectors (28) takes the form

$$
\left\langle\Psi_{N}(\mathbf{v}) \mid \Psi_{N}(\mathbf{u})\right\rangle=\sum_{\boldsymbol{\lambda} \subseteq\left\{(M+1-N)^{N}\right\}} S_{\boldsymbol{\lambda}}\left(\mathbf{v}^{-2}\right) S_{\boldsymbol{\lambda}}\left(\mathbf{u}^{2}\right)=\frac{\operatorname{det}\left(T_{k j}\right)_{1 \leq k, j \leq N}}{\mathcal{V}\left(\mathbf{u}^{2}\right) \mathcal{V}\left(\mathbf{v}^{-2}\right)} .
$$

In (29) we use the notation for the Vandermonde determinant,

$$
\mathcal{V}\left(\mathbf{u}^{2}\right) \equiv \prod_{1 \leq m<l \leq N}\left(u_{l}^{2}-u_{m}^{2}\right),
$$

while the entries $T_{k j}$ of the matrix of size $N \times N$ are of the form

$$
T_{k j}=\frac{1-\left(u_{k}^{2} / v_{j}^{2}\right)^{M+1}}{1-u_{k}^{2} / v_{j}^{2}} .
$$

Equations (29) and (31) are specifications of (26) and (27), respectively. On the solutions (12), the entries (31) are equal to $T_{j k}=(M+1) \delta_{j k}$, where the l'Hospital rule is taken into account on the principal diagonal. We use the exponential parametrization for the solutions of the Bethe equations in a compact form:

$$
\mathbf{u}^{2}=e^{i \boldsymbol{\theta}}, \quad e^{i \boldsymbol{\theta}} \equiv\left(e^{i \theta_{1}}, e^{i \theta_{2}}, \ldots, e^{i \theta_{N}}\right),
$$

where the "angle" notation (12) or (19) is meant. Thus, the answer for the squared norm $\mathcal{N}^{2}(\boldsymbol{\theta}) \equiv\left\langle\Psi_{N}(\boldsymbol{\theta}) \mid \Psi_{N}(\boldsymbol{\theta})\right\rangle$ of the Bethe eigenvector (28) looks like

$$
\mathcal{N}^{2}(\boldsymbol{\theta})=\frac{(M+1)^{N}}{\mathcal{V}\left(e^{i \boldsymbol{\theta}}\right) \mathcal{V}\left(e^{-i \boldsymbol{\theta}}\right)}=\frac{(M+1)^{N}}{\prod_{1 \leq m<l \leq N} 2\left(1-\cos \left(\theta_{l}-\theta_{m}\right)\right)} .
$$

Now we turn to the ratio

$$
\mathcal{T}(\mathbf{v}, \mathbf{u}, n) \equiv \frac{\left\langle\Psi_{N}(\mathbf{v})\left|\bar{\Pi}_{n}\right| \Psi_{N}(\mathbf{u})\right\rangle}{\mathcal{N}(\mathbf{v}) \mathcal{N}(\mathbf{u})}
$$

where the projector $\bar{\Pi}_{n}$ is defined in (25), and $\mathcal{N}^{2}(\mathbf{u})=\left\langle\Psi_{N}(\mathbf{u}) \mid \Psi_{N}(\mathbf{u})\right\rangle$ for an arbitrary parametrization of the state vectors. Using (28), we calculate:

$$
\bar{\Pi}_{n}\left|\Psi_{N}(\mathbf{u})\right\rangle=\sum_{\boldsymbol{\lambda} \subseteq\left\{(M-N-n)^{N}\right\}} S_{\boldsymbol{\lambda}}\left(\mathbf{u}^{2}\right)\left(\prod_{k=M-n}^{M}\left(\sigma_{k}^{-}\right)^{0}\right)\left(\prod_{k=0}^{M-n-1}\left(\sigma_{k}^{-}\right)^{e_{k}}\right)|\uparrow\rangle,
$$


where the summation goes over the nonstrict partitions $\boldsymbol{\lambda}$ respecting the condition $M-$ $N-n \geq \lambda_{1} \geq \lambda_{2} \geq \cdots \geq \lambda_{N} \geq 0$. Taking (28) and (35) into account, and using the Binet-Cauchy formula, we calculate the numerator in (34):

$$
\begin{aligned}
\left\langle\Psi_{N}(\mathbf{v})\left|\bar{\Pi}_{n}\right| \Psi_{N}(\mathbf{u})\right\rangle & =\sum_{\boldsymbol{\lambda} \subseteq\left\{(M-N-n)^{N}\right\}} S_{\boldsymbol{\lambda}}\left(\mathbf{v}^{-2}\right) S_{\boldsymbol{\lambda}}\left(\mathbf{u}^{2}\right) \\
& =\frac{1}{\mathcal{V}\left(\mathbf{u}^{2}\right) \mathcal{V}\left(\mathbf{v}^{-2}\right)} \operatorname{det}\left(\frac{1-\left(u_{k}^{2} / v_{j}^{2}\right)^{M-n}}{1-u_{k}^{2} / v_{j}^{2}}\right)_{1 \leq j, k \leq N} .
\end{aligned}
$$

Assume that the sets of parameters $\mathbf{v}$ and $\mathbf{u}$ in (34) coincide and consist of the solutions (12). Then, the expression $\mathcal{T}(\boldsymbol{\theta}, n) \equiv \mathcal{T}\left(e^{i \boldsymbol{\theta} / 2}, e^{i \boldsymbol{\theta} / 2}, n\right)$ is related to what is called the emptiness formation probability, which provides the probability of formation of a string of the empty (i.e., spin "up") states on the last $n+1$ sites of the lattice. Eventually, formula (36) leads to $\mathcal{T}(\boldsymbol{\theta}, n)$ in the same determinantal form as in [41, 42, (see therein for more references):

$$
\mathcal{T}(\boldsymbol{\theta}, n)=\operatorname{det}\left(\left(1-\frac{n+1}{M+1}\right) \delta_{j k}+\frac{1-e^{i\left(\theta_{j}-\theta_{k}\right)(n+1)}}{(M+1)\left(1-e^{i\left(\theta_{k}-\theta_{j}\right)}\right)}\left(1-\delta_{j k}\right)\right)_{1 \leq j, k \leq N},
$$

where the parameters $\theta_{l}, 1 \leq l \leq N$, correspond to the parametrization (12).

2.2. Thermal correlator of the ferromagnetic string and random walks of vicious walkers. We turn to a calculation of the following expectation value:

$$
\mathcal{T}(\mathbf{v}, \mathbf{u}, n, \beta) \equiv \frac{\left\langle\Psi_{N}(\mathbf{v})\left|\bar{\Pi}_{n} e^{-\beta \widehat{H}_{X X}} \bar{\Pi}_{n}\right| \Psi_{N}(\mathbf{u})\right\rangle}{\mathcal{N}(\mathbf{v}) \mathcal{N}(\mathbf{u})}
$$

which clearly reduces to $\mathcal{T}(\mathbf{v}, \mathbf{u}, n)$ (see (34)) at $\beta=0$. However, our aim is to obtain $\mathcal{T}(\boldsymbol{\theta}, n, \beta)$ (see (25)) simply by taking the arguments $\mathbf{u}$ and $\mathbf{v}$ in (38) that coincide with one and the same solution (12).

We use the technique presented above to calculate the numerator in (38). Using formula (35) (and its conjugate), we obtain

$$
\begin{aligned}
& \left\langle\Psi_{N}(\mathbf{v})\left|\bar{\Pi}_{n} e^{-\beta \widehat{H}_{X X}} \bar{\Pi}_{n}\right| \Psi_{N}(\mathbf{u})\right\rangle \\
& \quad=\sum_{\boldsymbol{\lambda}^{L}, \boldsymbol{\lambda}^{R} \subseteq\left\{(M-N-n)^{N}\right\}} S_{\boldsymbol{\lambda}^{L}}\left(\mathbf{v}^{-2}\right) S_{\boldsymbol{\lambda}^{R}}\left(\mathbf{u}^{2}\right) F_{\boldsymbol{\mu}^{L} ; \boldsymbol{\mu}^{R}}(\beta) .
\end{aligned}
$$

Summations in (39) run over the nonstrict partitions $\boldsymbol{\lambda}^{L}$ and $\boldsymbol{\lambda}^{R}$ of the same kind as in (35). The superscripts $L$ and $R$ are only to distinguish two independent summations. The corresponding strict partitions $\boldsymbol{\mu}^{L}$ and $\boldsymbol{\mu}^{R}$ are defined as follows: $\boldsymbol{\mu}^{L, R}=\boldsymbol{\lambda}^{L, R}+\boldsymbol{\delta}$, where $\boldsymbol{\delta} \equiv\left(\delta_{1}, \delta_{2}, \ldots, \delta_{N}\right), \delta_{j}=N-j$. The notation $F_{\boldsymbol{\mu}^{L} ; \boldsymbol{\mu}^{R}}(\beta)$ implies the following average:

$$
\begin{aligned}
F_{\boldsymbol{\mu}^{L} ; \boldsymbol{\mu}^{R}}(\beta) & \equiv F_{\mu_{1}^{L}, \mu_{2}^{L}, \ldots, \mu_{N}^{L} ; \mu_{1}^{R}, \mu_{2}^{R}, \ldots, \mu_{N}^{R}}(\beta) \\
& =\left\langle\Uparrow\left|\sigma_{\mu_{1}^{L}}^{+} \sigma_{\mu_{2}^{L}}^{+} \cdots \sigma_{\mu_{N}^{L}}^{+} e^{-\beta \widehat{H}_{X X}} \sigma_{\mu_{1}^{R}}^{-} \sigma_{\mu_{2}^{R}}^{-} \cdots \sigma_{\mu_{N}^{R}}^{-}\right| \Uparrow\right\rangle,
\end{aligned}
$$

which is none other than the $2 N$-point correlation function over the ferromagnetic state. It is related to enumeration of the admissible trajectories that are traced by $N$ vicious walkers traveling over sites of the one-dimensional chain 35]-38.

Indeed, let $\left|P_{K}\left(\mu_{1}^{R}, \ldots, \mu_{N}^{R} \rightarrow \mu_{1}^{L}, \ldots, \mu_{N}^{L}\right)\right|$ be the number of trajectories consisting of $K$ links made by $N$ vicious walkers in the random turns model. Here, the initial and final positions of the walkers on the sites are given, respectively, by elements of the strict decreasing partitions $\mu_{1}^{R}>\mu_{2}^{R}>\cdots>\mu_{N}^{R}$ and $\mu_{1}^{L}>\mu_{2}^{L}>\cdots>\mu_{N}^{L}$. We introduce the notation $\mathcal{D}_{\ell}^{K}$ for the operator of differentiation of order $K$ with respect to $\ell$ at the point 
$\ell=0$ [38]. Then, the application of the operator $\mathcal{D}_{\beta / 2}^{K}$ to the correlator (40) results in an average of the type

$$
\left\langle\Uparrow\left|\sigma_{\mu_{1}^{L}}^{+} \sigma_{\mu_{2}^{L}}^{+} \cdots \sigma_{\mu_{N}^{L}}^{+}\left(-2 \hat{H}_{X X}\right)^{K} \sigma_{\mu_{1}^{R}}^{-} \sigma_{\mu_{2}^{R}}^{-} \cdots \sigma_{\mu_{N}^{R}}^{-}\right| \Uparrow\right\rangle .
$$

In [36] it was established that this average leads to the numbers $\mid P_{K}\left(\mu_{1}^{R}, \ldots, \mu_{N}^{R} \rightarrow\right.$ $\left.\mu_{1}^{L}, \ldots, \mu_{N}^{L}\right) \mid$, with the help of the commutation relation

$$
\left[\widehat{H}_{X X}, \sigma_{\mu_{1}^{R}}^{-} \sigma_{\mu_{2}^{R}}^{-} \cdots \sigma_{\mu_{N}^{R}}^{-}\right]=\sum_{k=1}^{N} \sigma_{\mu_{1}^{R}}^{-} \cdots \sigma_{\mu_{k}^{R}-1}^{-}\left[\widehat{H}_{X X}, \sigma_{\mu_{k}^{R}}^{-}\right] \sigma_{\mu_{k}^{R}+1}^{-} \cdots \sigma_{\mu_{N}^{R}}^{-} .
$$

The nonintersection condition for trajectories of the walkers is expressed by the vanishing of the correlation function (40) for any pair of coinciding indices $\mu_{k}^{R}$ or $\mu_{p}^{L}$. Thus, we conclude that the average (39) turns out to be the generating function of the polynomials in $2 N$ variables $u_{1}^{2}, u_{2}^{2}, \ldots, u_{N}^{2}$ and $v_{1}^{-2}, v_{2}^{-2}, \ldots, v_{N}^{-2}$ :

$$
\begin{aligned}
\mathcal{D}_{\beta / 2}^{K} & {\left[\left\langle\Psi_{N}(\mathbf{v})\left|\bar{\Pi}_{n} e^{-\beta \widehat{H}_{X X}} \bar{\Pi}_{n}\right| \Psi_{N}(\mathbf{u})\right\rangle\right] } \\
& =\sum_{\boldsymbol{\lambda}^{L}, \boldsymbol{\lambda}^{R} \subseteq\left\{(M-N-n)^{N}\right\}}\left|P_{K}\left(\boldsymbol{\mu}^{R} \rightarrow \boldsymbol{\mu}^{L}\right)\right| S_{\boldsymbol{\lambda}^{L}}\left(\mathbf{v}^{-2}\right) S_{\boldsymbol{\lambda}^{R}}\left(\mathbf{u}^{2}\right)
\end{aligned}
$$

(recall that $\boldsymbol{\mu}^{L, R}=\boldsymbol{\lambda}^{L, R}+\boldsymbol{\delta}$ ). As was shown in [38, the number of trajectories of $K$ links traced by $N$ vicious walkers on an axis, i.e., $\left|P_{K}\left(\boldsymbol{\mu}^{R} \rightarrow \boldsymbol{\mu}^{L}\right)\right|$, is expressed through the number of trajectories of the same "length" $K$ that are traced by a single walker traveling over sites of the $N$-dimensional lattice of infinite extension.

The correlator (40) satisfies

$$
\begin{aligned}
& \frac{d}{d \beta} F_{\mu_{1}^{L}, \mu_{2}^{L}, \ldots, \mu_{N}^{L} ; \mu_{1}^{R}, \mu_{2}^{R}, \ldots, \mu_{N}^{R}}(\beta) \\
& =\frac{1}{2} \sum_{k=1}^{N}\left(F_{\mu_{1}^{L}, \mu_{2}^{L}, \ldots, \mu_{N}^{L} ; \mu_{1}^{R}, \mu_{2}^{R}, \ldots, \mu_{k}^{R}+1, \ldots, \mu_{N}^{R}}(\beta)+F_{\mu_{1}^{L}, \mu_{2}^{L}, \ldots, \mu_{N}^{L} ; \mu_{1}^{R}, \mu_{2}^{R}, \ldots, \mu_{k}^{R}-1, \ldots, \mu_{N}^{R}}(\beta)\right) .
\end{aligned}
$$

Equation (43) was considered in [36] in the case of the periodic boundary condition with respect to the lattice argument and with the initial condition

$$
F_{\mu_{1}^{L}, \mu_{2}^{L}, \ldots, \mu_{N}^{L} ; \mu_{1}^{R}, \mu_{2}^{R}, \ldots, \mu_{N}^{R}}(0)=\prod_{k=1}^{N} \delta_{\mu_{k}^{L}, \mu_{k}^{R}} .
$$

The solution of (43) can be expressed as the determinant of the matrix $\left(F_{k ; l}(\beta)\right)_{1 \leq k, l \leq N}$ [35, 36]:

$$
F_{\mu_{1}^{L}, \mu_{2}^{L}, \ldots, \mu_{N}^{L} ; \mu_{1}^{R}, \mu_{2}^{R}, \ldots, \mu_{N}^{R}}(\beta)=\operatorname{det}\left(F_{\mu_{k}^{L} ; \mu_{l}^{R}}(\beta)\right)_{1 \leq k, l \leq N},
$$

where the entries satisfy the following difference-differential equation:

$$
\frac{d}{d \beta} F_{k ; l}(\beta)=\frac{1}{2}\left(F_{k+1 ; l}(\beta)+F_{k-1 ; l}(\beta)\right) .
$$

A similar equation can also be obtained for the fixed index $l$.

It can be checked that the transition amplitude $\left\langle\Uparrow\left|\sigma_{k}^{+} e^{-\beta \widehat{H}_{X X}} \sigma_{l}^{-}\right| \Uparrow\right\rangle$ respects (45); see 35-38. This average can be viewed as the generating function of the number of walks with random turns of a single pedestrian traveling between the $l$ th and the $k$ th sites of a (periodic) chain [36, 38. The solution of (45) can be written as the sum

$$
F_{k ; l}(\beta) \equiv \frac{1}{M+1} \sum_{s=0}^{M} e^{\beta \cos \phi_{s}} e^{i \phi_{s}(k-l)},
$$


where the parametrization $\phi_{s}=\frac{2 \pi}{M+1}\left(s-\frac{M}{2}\right)$ is used. The periodicity condition with respect of the lattice argument and the "initial" condition $F_{k ; l}(0)=\delta_{k, l}$ are imposed.

Using (44) and (46), we reexpress (40) in terms of the Schur functions (22) and the Vandermonde determinants (30) as follows [35]:

$$
\begin{aligned}
& F_{\boldsymbol{\mu}^{L} ; \boldsymbol{\mu}^{R}}(\beta)=\frac{1}{(M+1)^{N}} \sum_{M \geq k_{1}>k_{2}>\cdots>k_{N} \geq 0} e^{\beta \sum_{l=1}^{N} \cos \left(\phi_{k_{l}}\right)} \\
& \times \mathcal{V}\left(e^{i \phi}\right) \mathcal{V}\left(e^{-i \phi}\right) S_{\boldsymbol{\lambda}^{L}}\left(e^{i \phi}\right) S_{\boldsymbol{\lambda}^{R}}\left(e^{-i \phi}\right) .
\end{aligned}
$$

The parametrization $\phi_{s}, 0 \leq s \leq M$, used in (47), is the same as in (46). We continue to use boldface letters to denote $N$-tuples of numbers: for instance, $\phi$ corresponds to $\left(\phi_{k_{1}}, \phi_{k_{2}}, \ldots, \phi_{k_{N}}\right)$. We substitute (47) in (39) and use (36) to calculate sums:

$$
\mathcal{P}\left(\mathbf{v}^{-2}, e^{i \phi}\right) \equiv \sum_{\boldsymbol{\lambda}^{L}} S_{\boldsymbol{\lambda}^{L}}\left(\mathbf{v}^{-2}\right) S_{\boldsymbol{\lambda}^{L}}\left(e^{i \phi}\right), \quad \mathcal{P}\left(e^{-i \phi}, \mathbf{u}^{2}\right) \equiv \sum_{\boldsymbol{\lambda}^{R}} S_{\boldsymbol{\lambda}^{R}}\left(e^{-i \phi}\right) S_{\boldsymbol{\lambda}^{R}}\left(\mathbf{u}^{2}\right) .
$$

The range of summation in (48) is taken as follows: $\boldsymbol{\lambda}^{L}, \boldsymbol{\lambda}^{R} \subseteq\left\{(M-N-n)^{N}\right\}$. Then we obtain

$$
\begin{aligned}
\left\langle\Psi_{N}(\mathbf{v})\right| & \bar{\Pi}_{n} e^{-\beta \widehat{H}_{X X}} \bar{\Pi}_{n}\left|\Psi_{N}(\mathbf{u})\right\rangle \\
= & \frac{1}{(M+1)^{N} \mathcal{V}\left(\mathbf{u}^{2}\right) \mathcal{V}\left(\mathbf{v}^{-2}\right)} \sum_{M \geq k_{1}>k_{2}>\cdots>k_{N} \geq 0} e^{\beta \sum_{l=1}^{N} \cos \left(\phi_{k_{l}}\right)} \\
& \quad \times \operatorname{det}\left(\frac{1-\left(e^{i \phi_{k_{i}} v_{j}^{-2}}\right)^{M-n}}{1-e^{i \phi_{k_{i}}} v_{j}^{-2}}\right)_{1 \leq i, j \leq N} \operatorname{det}\left(\frac{1-\left(u_{p}^{2} e^{-i \phi_{k_{l}}}\right)^{M-n}}{1-u_{p}^{2} e^{-i \phi_{k_{l}}}}\right)_{1 \leq p, l \leq N} .
\end{aligned}
$$

The Binet-Cauchy formula enables us to evaluate (49) as follows:

$$
\begin{aligned}
& \left\langle\Psi_{N}(\mathbf{v})\left|\bar{\Pi}_{n} e^{-\beta \widehat{H}_{X X}} \bar{\Pi}_{n}\right| \Psi_{N}(\mathbf{u})\right\rangle \\
& =\frac{1}{\mathcal{V}\left(\mathbf{u}^{2}\right) \mathcal{V}\left(\mathbf{v}^{-2}\right)} \operatorname{det}\left(\sum_{k, l=0}^{M-n-1} F_{k ; l}(\beta) \frac{u_{i}^{2 l}}{v_{j}^{2 k}}\right)_{1 \leq i, j \leq N},
\end{aligned}
$$

where $F_{k ; l}(\beta)$ is defined by (46). Clearly, relation (50) at $\beta=0$ reduces to (36). The expression for $\mathcal{T}(\boldsymbol{\theta}, n, \beta)$ (see (25)) can be obtained straightforwardly from (50) with coinciding parameters $\mathbf{u}=\mathbf{v}$ (which are solutions of the Bethe equations) with the help of $\mathcal{N}^{2}$ (see (33)) :

$$
\mathcal{T}(\boldsymbol{\theta}, n, \beta)=\frac{1}{(M+1)^{N}} \operatorname{det}\left(\sum_{k, l=0}^{M-n-1} F_{k ; l}(\beta) e^{i\left(l \theta_{i}-k \theta_{j}\right)}\right)_{1 \leq i, j \leq N} .
$$

\section{§3. Strongly anisotropic XXZ Chain}

3.1. The Bethe state vectors and their form factors. We turn to the strong anisotropy limit, $\Delta \rightarrow-\infty$, which is described by the Hamiltonian $\hat{H}_{\mathrm{SA}}$ (see (15)). The corresponding state vector is given by equation (3),

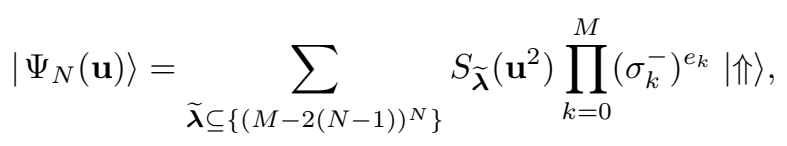

where $S_{\widetilde{\boldsymbol{\lambda}}}\left(\mathbf{u}^{2}\right)$ is as in (24), and the summation goes over all nonstrict partitions. Summation over the strict partitions $\boldsymbol{\mu}$ with elements respecting the condition $\mu_{i}>\mu_{i+1}+1$ is 
equivalent to that over the nonstrict partitions $\tilde{\boldsymbol{\lambda}}$, where $\tilde{\boldsymbol{\lambda}}=\boldsymbol{\mu}-2 \boldsymbol{\delta}$ and $M+2(1-N) \geq$ $\widetilde{\lambda}_{1} \geq \widetilde{\lambda}_{2} \geq \cdots \geq \tilde{\lambda}_{N} \geq 0$. The scalar product of the state vector (51) by its conjugate (defined much as in (28)) is given by the relation looking, in its turn, like (29):

$$
\left\langle\Psi_{N}(\mathbf{v}) \mid \Psi_{N}(\mathbf{u})\right\rangle=\sum_{\widetilde{\boldsymbol{\lambda}} \subseteq\left\{(M-2(N-1))^{N}\right\}} S_{\widetilde{\boldsymbol{\lambda}}}\left(\mathbf{v}^{-2}\right) S_{\widetilde{\boldsymbol{\lambda}}}\left(\mathbf{u}^{2}\right)=\frac{\operatorname{det}\left(T_{k j}\right)_{1 \leq k, j \leq N}}{\mathcal{V}\left(\mathbf{u}^{2}\right) \mathcal{V}\left(\mathbf{v}^{-2}\right)},
$$

where the entries $T_{k j}$ take the form:

$$
T_{k j}=\frac{1-\left(u_{k}^{2} / v_{j}^{2}\right)^{M-N+2}}{1-u_{k}^{2} / v_{j}^{2}},
$$

and the notation (30) for the Vandermonde determinant is used. When the sets of parameters $\mathbf{v}$ and $\mathbf{u}$ on the left-hand side of (52) coincide, the Bethe equations (18) allow us to express the entries (53) as follows: $T_{j k}=1+(M-N+1) \delta_{j k}$. Therefore, the norm of the Bethe eigenvectors $\mathcal{N}^{2}(\boldsymbol{\theta}) \equiv\left\langle\Psi_{N}(\boldsymbol{\theta}) \mid \Psi_{N}(\boldsymbol{\theta})\right\rangle$ is given by

$$
\mathcal{N}^{2}(\boldsymbol{\theta})=\frac{(M+1)(M+1-N)^{N-1}}{\mathcal{V}\left(e^{i \boldsymbol{\theta}}\right) \mathcal{V}\left(e^{-i \boldsymbol{\theta}}\right)}=\frac{(M+1)(M+1-N)^{N-1}}{\prod_{1 \leq m<l \leq N} 2\left(1-\cos \left(\theta_{l}-\theta_{m}\right)\right)} .
$$

The exponential parametrization (19) is used in (54) in the compact form (32). It can be shown that the scalar product $\left\langle\Psi_{N}(\mathbf{v}) \mid \Psi_{N}(\mathbf{u})\right\rangle$ (see (52)) vanishes (i.e., the state vectors are orthogonal) provided the parameters $\mathbf{u}$ and $\mathbf{v}$ are independent Bethe solutions.

The numerator of the ratio (34) is calculated in the same way as in Subsection 2.1, i.e., with the help of the Binet-Cauchy formula:

$$
\begin{aligned}
\left\langle\Psi_{N}(\mathbf{v})\left|\bar{\Pi}_{n}\right| \Psi_{N}(\mathbf{u})\right\rangle & =\sum_{\widetilde{\boldsymbol{\lambda}} \subseteq\left\{(M-2 N-n+1)^{N}\right\}} S_{\widetilde{\boldsymbol{\lambda}}}\left(\mathbf{v}^{-2}\right) S_{\widetilde{\boldsymbol{\lambda}}}\left(\mathbf{u}^{2}\right) \\
& =\frac{1}{\mathcal{V}\left(\mathbf{u}^{2}\right) \mathcal{V}\left(\mathbf{v}^{-2}\right)} \operatorname{det}\left(\frac{1-\left(u_{k}^{2} / v_{j}^{2}\right)^{M-N-n+1}}{1-u_{k}^{2} / v_{j}^{2}}\right)_{1 \leq j, k \leq N} .
\end{aligned}
$$

After using (54) and (55), the answer for (34), taken on the solutions of the Bethe equation (19) (i.e., the emptiness formation probability), takes the following form:

$$
\begin{aligned}
\mathcal{T}(\boldsymbol{\theta}, n)=\frac{M-N+1}{M+1} \times \operatorname{det} & ( \\
& \left(1-\frac{n}{M-N+1}\right) \delta_{j k} \\
& \left.+\frac{1-e^{i n\left(\theta_{j}-\theta_{k}\right)}}{(M-N+1)\left(1-e^{i\left(\theta_{k}-\theta_{j}\right)}\right)}\left(1-\delta_{j k}\right)\right)_{1 \leq k, j \leq N} .
\end{aligned}
$$

3.2. Thermal correlator of ferromagnetic string. We proceed to a calculation of the average $\mathcal{T}(\mathbf{v}, \mathbf{u}, n, \beta)$ (see (38) ), where the Hamiltonian $\widehat{H}_{X X}$ is replaced by $\widehat{H}_{\mathrm{SA}}$ (see (15)). Using formula (51) (and its conjugate), we obtain the corresponding numerator of (38):

$$
\begin{aligned}
& \left\langle\Psi_{N}(\mathbf{v})\left|\bar{\Pi}_{n} e^{-\beta \widehat{H}_{\mathrm{SA}}} \bar{\Pi}_{n}\right| \Psi_{N}(\mathbf{u})\right\rangle \\
& \quad=\sum_{\widetilde{\boldsymbol{\lambda}}^{L}, \widetilde{\boldsymbol{\lambda}}^{R} \subseteq\left\{(M-2 N-n+1)^{N}\right\}} S_{\widetilde{\boldsymbol{\lambda}}^{L}}\left(\mathbf{v}^{-2}\right) S_{\widetilde{\boldsymbol{\lambda}}^{R}}\left(\mathbf{u}^{2}\right) F_{\widetilde{\boldsymbol{\mu}}^{L} ; \widetilde{\boldsymbol{\mu}}^{R}}(\beta),
\end{aligned}
$$

where $F_{\widetilde{\boldsymbol{\mu}}^{L} ; \tilde{\boldsymbol{\mu}}^{R}}(\beta)$ is the $2 N$-point correlation function over the ferromagnetic state given, practically, by (40), except that $\widehat{H}_{X X}$ as in (8) is replaced by $\widehat{H}_{\mathrm{SA}}$. The summations in (57) go over the nonstrict partitions $\widetilde{\boldsymbol{\lambda}}^{L}$ and $\widetilde{\boldsymbol{\lambda}}^{R}$ of the same kind as, e.g., in (52). The corresponding strict partitions $\widetilde{\boldsymbol{\mu}}^{L}$ and $\widetilde{\boldsymbol{\mu}}^{R}$ are defined as $\widetilde{\boldsymbol{\mu}}^{L, R}=\widetilde{\boldsymbol{\lambda}}^{L, R}+2 \boldsymbol{\delta}$, where $\boldsymbol{\delta}$ denotes the partition $(N-1, N-2, \ldots, 0)$. It is crucial that now the lattice indices $\widetilde{\boldsymbol{\mu}}^{L}$, 
$\widetilde{\boldsymbol{\mu}}^{R}$ respect the exclusion requirement: occupation of nearest sites is forbidden. Moreover, an analog of (42) can be written as follows:

$$
\begin{aligned}
\mathcal{D}_{\beta / 2}^{K} & {\left[\left\langle\Psi_{N}(\mathbf{v})\left|\bar{\Pi}_{n} e^{-\beta \widehat{H}_{\mathrm{SA}}} \bar{\Pi}_{n}\right| \Psi_{N}(\mathbf{u})\right\rangle\right] } \\
& =\sum_{\widetilde{\boldsymbol{\lambda}}^{L}, \widetilde{\boldsymbol{\lambda}}^{R} \subseteq\left\{(M-2 N-n+1)^{N}\right\}}\left|P_{K}\left(\widetilde{\boldsymbol{\mu}}^{R} \rightarrow \widetilde{\boldsymbol{\mu}}^{L}\right)\right| S_{\widetilde{\boldsymbol{\lambda}}^{L}}\left(\mathbf{v}^{-2}\right) S_{\widetilde{\boldsymbol{\lambda}}^{R}}\left(\mathbf{u}^{2}\right) .
\end{aligned}
$$

The solutions of the Bethe equation (18) provide a complete set of eigenstates [52]. Taking the orthogonality of the corresponding states into account, we can arrive at resolution of the identity operator:

$$
\mathbb{I}=\sum_{\{\boldsymbol{\theta}\}} \mathcal{N}^{-2}(\boldsymbol{\theta})\left|\Psi_{N}(\boldsymbol{\theta})\right\rangle\left\langle\Psi_{N}(\boldsymbol{\theta})\right|,
$$

where the summation is over all independent solutions of the Bethe equation (18), and the squared norm $\mathcal{N}^{2}(\boldsymbol{\theta})$ is given by (54). We shall calculate (57), inserting (59) in the left-hand side of (57) and applying (55). We employ the formula

$$
\left\langle\Psi_{N}\left(\mathbf{v}^{\prime}\right)\left|e^{-\beta \widehat{H}_{\mathrm{SA}}}\right| \Psi_{N}(\boldsymbol{\theta})\right\rangle=\left\langle\Psi_{N}\left(\mathbf{v}^{\prime}\right) \mid \Psi_{N}(\boldsymbol{\theta})\right\rangle e^{-\beta E_{N}(\boldsymbol{\theta})},
$$

where the energy $E_{N}(\boldsymbol{\theta})$ is given by (21). Using (55), finally we obtain

$$
\begin{aligned}
& \left\langle\Psi_{N}(\mathbf{v})\left|\bar{\Pi}_{n} e^{-\beta \widehat{H}_{\mathrm{SA}}} \bar{\Pi}_{n}\right| \Psi_{N}(\mathbf{u})\right\rangle \\
& \quad=\frac{1}{(M+1)(M+1-N)^{N-1} \mathcal{V}\left(\mathbf{u}^{2}\right) \mathcal{V}\left(\mathbf{v}^{-2}\right)} \sum_{M-N \geq I_{1}>I_{2}>\cdots>I_{N} \geq 0} e^{\beta E_{N}(\boldsymbol{\theta})} \\
& \quad \times \operatorname{det}\left(\frac{1-\left(e^{i \theta_{i}} v_{j}^{-2}\right)^{M-N-n+1}}{1-e^{i \theta_{i}} v_{j}^{-2}}\right)_{1 \leq i, j \leq N} \operatorname{det}\left(\frac{1-\left(u_{l}^{2} e^{-i \theta_{p}}\right)^{M-N-n+1}}{1-u_{l}^{2} e^{-i \theta_{p}}}\right)_{1 \leq l, p \leq N},
\end{aligned}
$$

where the summation is taken over the ordered sets $\left\{I_{k}\right\}_{1 \leq k \leq N}$ that parametrize solutions (19). The answer for $\mathcal{T}(\boldsymbol{\theta}, n, \beta)$ (see (25)) can be obtained with the help of (54) and (60), where we should put $\mathbf{u}^{2}=\mathbf{v}^{2}=e^{i \boldsymbol{\theta}}$, with $\boldsymbol{\theta}$ being the solution (20) for the ground state of the Bethe equations.

\section{$\S 4$. BOXED PLANE PARTitions}

We shall show that the scalar products of the state vectors and the emptiness formation probability are related to the generating functions of the boxed plane partitions.

An array $\left(\pi_{i, j}\right)_{i, j \geq 1}$ of nonnegative integers that are monotone nonincreasing as functions of both $i$ and $j(i, j \in\{1,2, \ldots\})$ is called a plane partition $\boldsymbol{\pi}$; see [55]. The integers $\pi_{i, j}$ are called the parts of the plane partition, and $|\boldsymbol{\pi}|=\sum_{i, j \geq 1} \pi_{i, j}$ is its volume. Each plane partition has a three-dimensional diagram which can be interpreted as a collection of stacks of unit cubes (three-dimensional Young diagram). The height of the stack with coordinates $(i, j)$ is equal to the part $\pi_{i, j}$ of the plane partition. If we have $i \leq r, j \leq s$, and $\pi_{i, j} \leq t$ for all cubes of a plane partition, we say that this plane partition is contained in a box with side lengths $r, s, t$. If $\pi_{i, j}>\pi_{i+1, j}$, i.e., if the parts of a plane partition $\boldsymbol{\pi}$ are decaying along each column, then $\boldsymbol{\pi}$ is called a column strict plane partition. A partition $\boldsymbol{\pi}$ that decays along each column and each row $\left(\pi_{i, j}>\pi_{i+1, j}\right.$ and $\left.\pi_{i, j}>\pi_{i, j+1}\right)$ will be called a strict plane partition. The element $\pi_{1,1}$ of a strict plane partition $\boldsymbol{\pi}$ satisfies the condition $\pi_{1,1} \geq 2 r-2$ if all $i, j$ do not exceed $r$.

An arbitrary plane partition in a box of size $r \times r \times t$ can be transformed into a column strict plane partition in a box of size $r \times r \times(t+r-1)$ by the following procedure: to 
an array $\left(\pi_{i, j}\right)_{i, j \geq 1}$, we add the $r \times r$ matrix

$$
\boldsymbol{\pi}_{\mathrm{cspp}}=\left(\begin{array}{cccc}
r-1 & r-1 & \cdots & r-1 \\
r-2 & r-2 & \cdots & r-2 \\
\vdots & \vdots & & \vdots \\
0 & 0 & \cdots & 0
\end{array}\right)
$$

which corresponds to a minimal column strict plane partition. The volumes of the column strict plane partition and the corresponding plane partition are related by the formula

$$
\left|\boldsymbol{\pi}_{\text {cspp }}\right|=|\boldsymbol{\pi}|+\frac{N^{2}(N-1)}{2} .
$$

An arbitrary plane partition in a box of size $r \times r \times t$ can be transformed into a strict plane partition in a box of size $r \times r \times(t+2 r-2)$ : to an array $\left(\pi_{i, j}\right)_{i, j \geq 1}$, we can add the $r \times r$ matrix

$$
\boldsymbol{\pi}_{\mathrm{spp}}=\left(\begin{array}{cccc}
2 r-2 & 2 r-3 & \cdots & r-1 \\
2 r-3 & 2 r-4 & \cdots & r-2 \\
\vdots & \vdots & & \vdots \\
r-1 & r-2 & \cdots & 0
\end{array}\right),
$$

which corresponds to a minimal strict plane partition. The volumes of the strict plane partition and the corresponding plane partition are related by the formula

$$
\left|\boldsymbol{\pi}_{\mathrm{spp}}\right|=|\boldsymbol{\pi}|+N^{2}(N-1) .
$$

The partition function of the three-dimensional Young diagrams, or in other words, the generating function of plane partitions is equal to

$$
Z(q)=\sum_{\{\boldsymbol{\pi}\}} q^{|\boldsymbol{\pi}|},
$$

where $q$ is a weight, and the summation is over all plane partitions in a box. Formulas (61) and (62) provide the relationship between partition functions of plane partitions of different types. The generating functions of the column strict and strict plane partitions placed into a box of size $N \times N \times M$ are equal to

$$
\begin{aligned}
& Z_{\mathrm{cspp}}(q)=q^{\frac{N^{2}}{2}(N-1)} \prod_{1 \leq j, k \leq N} \frac{1-q^{M+1+j-k}}{1-q^{j+k-1}}, \\
& Z_{\mathrm{spp}}(q)=q^{N^{2}(N-1)} \prod_{1 \leq j, k \leq N} \frac{1-q^{M+3-j-k}}{1-q^{j+k-1}},
\end{aligned}
$$

respectively.

The scalar product (29) is related to the partition function of the column strict threedimensional Young diagrams placed into an $N \times N \times M$ box. Indeed, the parametrizations $v_{j}=q^{-\frac{j}{2}}$ and $u_{j}=q^{\frac{j-1}{2}}$ give the following:

$$
\begin{aligned}
\left\langle\Psi_{N}\right. & \left(q^{-\frac{1}{2}}, \ldots, q^{-\frac{N}{2}}\right)\left|\Psi_{N}\left(1, \ldots, q^{\frac{N-1}{2}}\right)\right\rangle \\
& =\sum_{\boldsymbol{\lambda} \subseteq\left\{(M+1-N)^{N}\right\}} S_{\boldsymbol{\lambda}}\left(q, \ldots, q^{N}\right) S_{\boldsymbol{\lambda}}\left(1, \ldots, q^{N-1}\right) \\
& =\frac{1}{\mathcal{V}\left(q, \ldots, q^{N}\right) \mathcal{V}\left(1, \ldots, q^{N-1}\right)} \operatorname{det}\left(\frac{1-s^{j+k-1}}{1-q^{j+k-1}}\right)_{1 \leq j, k \leq N},
\end{aligned}
$$


where $s=q^{M+1}$, and

$$
\mathcal{V}^{-1}\left(q, \ldots, q^{N}\right) \mathcal{V}^{-1}\left(1, \ldots, q^{N-1}\right)=q^{-\frac{N}{6}(N-1)(2 N-1)} \prod_{1 \leq k<j \leq N}\left(1-q^{j-k}\right)^{-2} .
$$

The determinant in (66) was calculated in the paper [60] in connection with the alternating sign matrices enumeration problem:

$$
\begin{aligned}
& \operatorname{det}\left(\frac{1-s^{j+k-1}}{1-q^{j+k-1}}\right)_{1 \leq j, k \leq N} \\
& \quad=q^{\frac{N}{6}(N-1)(2 N-1)} \prod_{1 \leq k<j \leq N}\left(1-q^{j-k}\right)^{2} \prod_{k, j=1}^{N} \frac{1-s q^{j-k}}{1-q^{j+k-1}} .
\end{aligned}
$$

Using (64), (67), and (68), for (66) we obtain:

$$
\left\langle\Psi_{N}\left(q^{-\frac{1}{2}}, \ldots, q^{-\frac{N}{2}}\right) \mid \Psi_{N}\left(1, \ldots, q^{\frac{N-1}{2}}\right)\right\rangle=q^{-\frac{N^{2}}{2}(N-1)} Z_{\text {cspp }}(q) .
$$

Thus, formula (69) says that the scalar product of two state vectors coincides at $q=1$ with the number of column strict partitions in a box of size $N \times N \times M$, i.e., with $Z_{\text {cspp }}(1)$.

The same parametrizations $v_{j}=q^{-\frac{j}{2}}$ and $u_{j}=q^{\frac{j-1}{2}}$ enable us to express the scalar product (52) corresponding to the strong anisotropy limit. The same representation as before is valid, though now we have $s=q^{M-N+2}$, while the range of summation over $\widetilde{\boldsymbol{\lambda}}$ takes the form

$$
\widetilde{\boldsymbol{\lambda}} \subseteq\left\{(M-2(N-1))^{N}\right\} .
$$

Taking (68) into account, we obtain

$$
\left\langle\Psi_{N}\left(q^{-\frac{1}{2}}, \ldots, q^{-\frac{N}{2}}\right) \mid \Psi_{N}\left(1, \ldots, q^{\frac{N-1}{2}}\right)\right\rangle=q^{-N^{2}(N-1)} Z_{\text {spp }}(q),
$$

where $Z_{\mathrm{spp}}(q)$ is the generating function of the strict plane partitions (65). The corresponding value of the scalar product at $q=1$ coincides with the number of strict plane partitions $Z_{\mathrm{spp}}(1)$.

Now we turn to the expectation value of the ferromagnetic string (36). In the present parametrization, we can use (67) and (68) to get

$$
\begin{aligned}
& \left\langle\Psi_{N}\left(q^{-\frac{1}{2}}, \ldots, q^{-\frac{N}{2}}\right)\left|\bar{\Pi}_{n}\right| \Psi_{N}\left(1, \ldots, q^{\frac{N-1}{2}}\right)\right\rangle \\
& \quad=\sum_{\lambda \subseteq\left\{(M-N-n)^{N}\right\}} S_{\boldsymbol{\lambda}}\left(q, \ldots, q^{N}\right) S_{\boldsymbol{\lambda}}\left(1, \ldots, q^{N-1}\right)=\prod_{k, j=1}^{N} \frac{1-s q^{j-k}}{1-q^{j+k-1}},
\end{aligned}
$$

where $s=q^{M-n}$; i.e., (70) differs from (66) in the sense that $n+1$ is subtracted from $M+1$. The box containing the plane partitions is now of smaller maximal height: $N \times N \times(M-n-1)$. The expectation value on the left-hand side of (70), being viewed as $q \rightarrow 1$, "counts" the number of plane partitions of smaller height. The same is true for the case of strong anisotropy, and (55) takes the form

$$
\begin{aligned}
& \left\langle\Psi_{N}\left(q^{-\frac{1}{2}}, \ldots, q^{-\frac{N}{2}}\right)\left|\bar{\Pi}_{n}\right| \Psi_{N}\left(1, \ldots, q^{\frac{N-1}{2}}\right)\right\rangle \\
& \quad=\sum_{\tilde{\boldsymbol{\lambda}} \subseteq\left\{(M-2 N-n+1)^{N}\right\}} S_{\widetilde{\boldsymbol{\lambda}}}\left(q, \ldots, q^{N}\right) S_{\widetilde{\boldsymbol{\lambda}}}\left(1, \ldots, q^{N-1}\right)=\prod_{k, j=1}^{N} \frac{1-s q^{j-k}}{1-q^{j+k-1}},
\end{aligned}
$$

where $s=q^{M-N-n+1}$. 


\section{§5. LOW TEMPERATURE ASYMPTOTICS}

We pass to the case of sufficiently long $X X$-chains, i.e., $M \gg 1$ while $N$ is moderate: $M \gg N \gg 1$. Now the correlator $F_{k ; l}(\beta)$ is approximately given by the modified Bessel function instead of (46):

$$
F_{k ; l}(\beta)=I_{k-l}(\beta)=\frac{1}{2 \pi} \int_{-\pi}^{\pi} e^{\beta \cos \phi} e^{i(k-l) \phi} d \phi .
$$

In the limit of small "temperature" $(1 / \beta \rightarrow 0)$ and for moderate values of $m \equiv|k-l|$, we use the known asymptotics of the Bessel function, obtaining

$$
F_{k ; l}(\beta) \simeq \frac{e^{\beta}}{\sqrt{2 \pi \beta}}\left(1-\frac{4 m^{2}-1}{8 \beta}+\cdots\right)
$$

i.e., the power decay is governed by the critical exponent $\xi=-1 / 2$.

Since summations can be replaced by integrations for sufficiently large $M$, from (39) and (47) we get

$$
\begin{aligned}
& \left\langle\Psi_{N}(\mathbf{v})\left|\bar{\Pi}_{n} e^{-\beta \widehat{H}_{X X}} \bar{\Pi}_{n}\right| \Psi_{N}(\mathbf{u})\right\rangle \\
& \quad=\frac{e^{\beta N}}{N !} \prod_{i=1}^{N}\left(\int_{-\pi}^{\pi} \frac{d \phi_{i}}{2 \pi}\right) e^{-\beta \sum_{l=1}^{N}\left(1-\cos \phi_{l}\right)} \mathcal{P}\left(\mathbf{v}^{-2}, e^{i \phi}\right) \mathcal{P}\left(e^{-i \boldsymbol{\phi}}, \mathbf{u}^{2}\right) \prod_{1 \leq k<l \leq N}\left|e^{i \phi_{k}}-e^{i \phi_{l}}\right|^{2},
\end{aligned}
$$

where the continuous integration variables $\phi_{i} \in[0,2 \pi]$ replace the discrete variables $k_{i} \in \mathcal{M}$ in the following way: $\phi_{k_{i}} \mapsto \phi_{i}$, for all $i$. Next, as $\beta \rightarrow \infty$, the integral (74) can be approximated as follows:

$$
\begin{aligned}
\left\langle\Psi_{N}(\mathbf{v})\right| & \bar{\Pi}_{n} e^{-\beta \widehat{H}_{X X}} \bar{\Pi}_{n}\left|\Psi_{N}(\mathbf{u})\right\rangle \\
\simeq & \mathcal{P}\left(\mathbf{v}^{-2}, \mathbf{1}\right) \mathcal{P}\left(\mathbf{1}, \mathbf{u}^{2}\right) \frac{e^{\beta N}}{(2 \pi)^{N} N !} \\
& \times \int_{-\infty}^{\infty} \int_{-\infty}^{\infty} \cdots \int_{-\infty}^{\infty} e^{-(\beta / 2) \sum_{l=1}^{N} \phi_{l}^{2}} \prod_{1 \leq k<l \leq N}\left|\phi_{k}-\phi_{l}\right|^{2} d \phi_{1} d \phi_{2} \cdots d \phi_{N} .
\end{aligned}
$$

The boldface argument $\mathbf{1}$ in the sum, say $\mathcal{P}\left(\mathbf{1}, \mathbf{u}^{2}\right)$, implies that $S_{\boldsymbol{\lambda}}\left(e^{ \pm i \boldsymbol{\phi}}\right)$ in (48) is replaced by $S_{\boldsymbol{\lambda}}(1,1, \ldots, 1)$, provided that the $N$-tuple of exponentials $e^{ \pm i \phi}$ is replaced by the $N$-tuple of units. The corresponding value of the Schur function can be obtained 55]:

$$
S_{\boldsymbol{\lambda}}(1,1, \ldots, 1)=\frac{\prod_{1 \leq k<l \leq N}\left(\lambda_{l}-l-\lambda_{k}+k\right)}{(N-1) !(N-2) ! \cdots 1 ! 0 !} .
$$

The right-hand side of (76) coincides with the dimension $\mathrm{d}_{\boldsymbol{\lambda}} \equiv \operatorname{dim} \boldsymbol{\pi}_{\boldsymbol{\lambda}}$ of the unitary irreducible representations of the unitary group $\mathcal{U}(N)$ that corresponds to the signature of $\boldsymbol{\lambda}$, i.e., $\mathrm{d}_{\boldsymbol{\lambda}}=S_{\boldsymbol{\lambda}}(1,1, \ldots, 1)$ [57]. The Schur functions provide a basis of the ring of symmetric polynomials in $N$ variables 55 .

The integral in (75) is the Mehta integral [58] of the Gaussian unitary ensemble of random matrices. Its value is known, and the estimate for the right-hand side of (75) takes the form

$$
\left\langle\Psi_{N}(\mathbf{v})\left|\bar{\Pi}_{n} e^{-\beta \widehat{H}_{X X}} \bar{\Pi}_{n}\right| \Psi_{N}(\mathbf{u})\right\rangle \simeq \mathcal{P}\left(\mathbf{v}^{-2}, \mathbf{1}\right) \mathcal{P}\left(\mathbf{1}, \mathbf{u}^{2}\right) \frac{e^{\beta N}}{\beta^{N^{2} / 2}} \prod_{n=1}^{N} \frac{\Gamma(n)}{(2 \pi)^{1 / 2}} .
$$

We consider the case where $\mathbf{u}^{2}$ and $\mathbf{v}^{2}$ in (77) are solutions of the Bethe equations (10). The approximate estimates $\mathbf{u}^{2}=e^{i \boldsymbol{\theta}} \simeq \mathbf{1}$ and $\mathbf{v}^{-2}=e^{-i \boldsymbol{\theta}} \simeq \mathbf{1}$ are valid for $1 \ll N \ll M$. 
Thus, the limiting form of (77) looks like

$$
\begin{aligned}
& \left\langle\Psi_{N}(1, \ldots, 1)\left|\bar{\Pi}_{n} e^{-\beta \widehat{H}_{X X}} \bar{\Pi}_{n}\right| \Psi_{N}(1, \ldots, 1)\right\rangle \\
& \quad \simeq\left(\sum_{\boldsymbol{\lambda} \subseteq\left\{(M-N-n)^{N}\right\}} \mathrm{d}_{\boldsymbol{\lambda}} \mathrm{d}_{\boldsymbol{\lambda}}\right)^{2} \frac{e^{\beta N}}{\beta^{N^{2} / 2}} \prod_{n=1}^{N} \frac{\Gamma(n)}{(2 \pi)^{1 / 2}} .
\end{aligned}
$$

This estimate is proportional to the square of (70) at $q=1$, i.e., to the square of the number of boxed plane partitions of size $N \times N \times(M-n-1)$. As a result, the ratio of the average $\mathcal{T}(\boldsymbol{\theta}, n, \beta)$ (see (25)) to the partition function of the $X X$ model $\mathcal{Z}$ [39] can be estimated as follows:

$$
\frac{\mathcal{T}(\boldsymbol{\theta}, n, \beta)}{\mathcal{Z}} \simeq \text { const } \times\left(\prod_{k, j=1}^{N} \frac{M-n+j-k}{j+k-1}\right)^{2} \frac{e^{-\beta \mathcal{E}_{0}^{X X}}}{\beta^{N^{2} / 2}},
$$

where $\mathcal{E}_{0}^{X X}$ is the ground state energy of the $X X$ model. In the same limit, the generating function (42) is specified as follows:

$$
\begin{array}{r}
\mathcal{D}_{\beta / 2}^{K}\left[\left\langle\Psi_{N}(1, \ldots, 1)\left|\bar{\Pi}_{n} e^{-\beta \widehat{H}_{X X}} \bar{\Pi}_{n}\right| \Psi_{N}(1, \ldots, 1)\right\rangle\right] \\
=\sum_{\boldsymbol{\lambda}^{L}, \boldsymbol{\lambda}^{R} \subseteq\left\{(M-N-n)^{N}\right\}}\left|P_{K}\left(\boldsymbol{\mu}^{R} \rightarrow \boldsymbol{\mu}^{L}\right)\right| \mathrm{d}_{\boldsymbol{\lambda}^{L}} \mathrm{~d}_{\boldsymbol{\lambda}^{R}},
\end{array}
$$

where the strict and nonstrict partitions are related by $\boldsymbol{\mu}^{L, R}=\boldsymbol{\lambda}^{L, R}+\boldsymbol{\delta}$ (see, e.g., (39))

In the case of strong anisotropy, we can use (71) to obtain a similar estimate that demonstrates the proportionality to the square of the number of strict plane partitions in a box of size $N \times N \times(M-n-N)$ :

$$
\begin{aligned}
\frac{\mathcal{T}(\boldsymbol{\theta}, n, \beta)}{\mathcal{Z}} & \simeq \operatorname{const} \times\left(\sum_{\tilde{\boldsymbol{\lambda}} \subseteq\left\{(M-2 N-n+1)^{N}\right\}} \mathrm{d}_{\tilde{\boldsymbol{\lambda}}} \mathrm{d}_{\tilde{\boldsymbol{\lambda}}}\right)^{2} \frac{e^{-\beta \mathcal{E}_{0}^{\mathrm{SA}}}}{\beta^{N^{2} / 2}} \\
& =\operatorname{const} \times\left(\prod_{k, j=1}^{N} \frac{M-N+1-n+j-k}{j+k-1}\right)^{2} \frac{e^{-\beta \mathcal{E}_{0}^{\mathrm{SA}}}}{\beta^{N^{2} / 2}},
\end{aligned}
$$

where $\mathcal{Z}$ is the partition function of the model in the strong anisotropy limit [5, 8], and $\mathcal{E}_{0}^{\text {inf }}$ is the corresponding ground state energy. Relation (58) is specified as follows:

$$
\begin{aligned}
\mathcal{D}_{\beta / 2}^{K} & {\left[\left\langle\Psi_{N}(1, \ldots, 1)\left|\bar{\Pi}_{n} e^{-\beta \widehat{H}_{\mathrm{SA}}} \bar{\Pi}_{n}\right| \Psi_{N}(1, \ldots, 1)\right\rangle\right] } \\
& =\sum_{\widetilde{\boldsymbol{\lambda}}^{L}, \widetilde{\boldsymbol{\lambda}}^{R} \subseteq\left\{(M-2 N-n+1)^{N}\right\}}\left|P_{K}\left(\widetilde{\boldsymbol{\mu}}^{R} \rightarrow \widetilde{\boldsymbol{\mu}}^{L}\right)\right| \mathrm{d}_{\tilde{\boldsymbol{\lambda}}^{L}} \mathrm{~d}_{\widetilde{\boldsymbol{\lambda}}^{R}},
\end{aligned}
$$

where $\widetilde{\boldsymbol{\mu}}^{L, R}=\widetilde{\boldsymbol{\lambda}}^{L, R}+2 \boldsymbol{\delta}($ see (57) $)$.

\section{$\S 6$. Discussion}

The $X X Z$ Heisenberg chain has been considered for two specific limits of the anisotropy parameter: $\Delta \rightarrow 0$ and $\Delta \rightarrow-\infty$. The corresponding state vectors are expressed in terms of symmetric Schur functions. Certain expectation values and thermal correlation functions of the ferromagnetic string operators have been calculated over the basis of $N$-particle Bethe states. The expectation values obtained are of the type of the emptiness formation probability. The thermal correlator of the ferromagnetic string operator is expressed through the generating function of the lattice paths of random walks of vicious walkers. The thermal correlator in question turns out to be the generating function of certain polynomials built up from the Schur polynomials. A relationship between the 
expectation values obtained and the generating functions of boxed plane partitions is discussed. An asymptotic estimate of the thermal expectation value of the ferromagnetic string is obtained in the limit of zero temperature for $\Delta=0$. These estimates are expressed in terms of the dimension of the irreducible representations of the group $\mathcal{U}(N)$.

\section{REFERENCES}

[1] W. Heisenberg, Zur Theorie des Ferromagnetismus, Z. Phys. 49 (1928), no. 9-10, 619-636.

[2] H. Bethe, Zur Theorie der Metalle. I. Eigenwerte und Eigenfunktionen Atomkette, Z. Phys. 71 (1931), no. 3-4, 205-226.

[3] C. N. Yang and C. P. Yang, One-dimensional chain of anisotropic spin-spin interactions. I. Proof of Bethe's hypothesis for ground state in a finite system, Phys. Rev. 150 (1966), no. 1, 321-327.

[4] , One-dimensional chain of anisotropic spin-spin interactions. II. Properties of the ground state energy per lattice site for an infinite system, Phys. Rev. 150 (1966), no. 1, 327-339.

[5] _ One-dimensional chain of anisotropic spin-spin interactions. III. Applications, Phys. Rev. 151 (1966), no. 1, 258-264.

[6] E. H. Lieb and F. Y. Wu, Two dimensional ferroelectric models, Phase Transitions and Critical Phenomena. Vol. 1 (C. Domb, M. Green, eds.), Acad. Press, London, 1972, pp. 331-490.

[7] R. J. Baxter, Exactly solved models in statistical mechanics, Acad. Press, London, 1982. MR0690578 (86i:82002a)

[8] M. Gaudin, La fonction d'onde de Bethe, Masson, Paris, 1983. MR0693905 (85h:82001)

[9] L. D. Faddeev, Quantum completely integrable models of field theory, Mathematical Physics Reviews. Vol. 1, Sov. Sci. Rev. Sect. C: Math. Phys. Rev., vol. 1, Harwood Acad. Publ., Chur, 1980, pp. 107-155; 40 years in mathematical physics, World Sci. Ser. 20th Century Math., vol. 2, World Sci., Singapore, 1995, pp. 187-235. MR0659260 (83e: 81002a) MR.1372058 (97c: 01048)

[10] P. P. Kulish and E. K. Sklyanin, Quantum spectral transform method. Recent developments, Lecture Notes in Phys., vol. 151, Springer, Berlin, 1982, pp. 61-119. MR0671263 (84m:81114)

[11] L. D. Faddeev and L. A. Takhtadzhyan, The quantum method for the inverse problem and the $X Y Z$ Heisenberg model, Uspekhi Mat. Nauk 34 (1979), no. 5, 13-63. (Russian) MR0562799 (81d:82066)

[12] W What is the spin of a spin wave? Phys. Lett. A 85 (1981), no. 6-7, 375-377. MR0632380 (83b:82017)

[13] V. E. Korepin, Calculation of norms of Bethe wave functions, Comm. Math. Phys. 86 (1982), no. 3, 391-418. MR0677006 (83m:81078)

[14] A. G. Izergin and V. E. Korepin, Correlation functions for the Heisenberg XXZ-antiferromagnet, Comm. Math. Phys. 99 (1985), no. 2, 271-302. MR0790738 (86m:82021)

[15] P. P. Kulish and F. A. Smirnov, Anisotropic Heisenberg ferromagnet with a ground of the domain wall type, J. Phys. C 18 (1985), no. 5, 1037-1048.

[16] N. M. Bogolyubov, A. G. Izergin, and V. E. Korepin, Correlation functions of integrable systems and the quantum inverse problem method, Nauka, Moscow, 1992. (Russian) MR1225798 (95j:81240)

[17] _ Quantum inverse scattering method and correlation functions, Cambridge Univ. Press, Cambridge, 1993. MR1245942 (95b:81224)

[18] F. H. L. Eßler, H. Frahm, A. G. Izergin, and V. E. Korepin, Determinant representation for correlation functions of spin-1/2 XXX and $X X Z$ Heisenberg magnets, Comm. Math. Phys. 174 (1995), no. 1, 191-214. MR1372806 (96j:82013)

[19] N. Kitanine, J. M. Maillet, N. Slavnov, and V. Terras, Spin-spin correlation functions of the XXZ$\frac{1}{2}$ Heisenberg chain in a magnetic field, Nuclear Phys. B 641 (2002), no. 3, 487-518. MR1931297 (2003h:82033)

[20] Correlation functions of the XXZ spin- $\frac{1}{2}$ Heisenberg chain at the free fermion point from their multiple integral representations, Nuclear Phys. B 642 (2002), no. 3, 433-455. MR1932594 (2003h:82022)

[21] M. E. Fisher, Walks, walls, wetting, and melting, J. Statist. Phys. 34 (1984), no. 5-6, 667-729. MR0751710 (85j:82022)

[22] P. J. Forrester, Exact solution of the lock step model of vicious walkers, J. Phys. A 23 (1990), no. 7, 1259-1273. MR1049530 (91d:82019)

[23] T. Nagao and P. J. Forrester, Vicious random walkers and a discretization of Gaussian random matrix ensembles, Nuclear Phys. B 620 (2002), no. 3, 551-565. MR1877962 (2002j:82056)

[24] A. J. Guttmann, A. L. Owczarek, and X. G. Viennot, Vicious walkers and Young tableaux. I. Without walls, J. Phys. A 31 (1998), no. 40, 8123-8135. MR1651492(99j:82042)

[25] C. Krattenthaler, A. J. Guttmann, and X. G. Viennot, Vicious walkers, friendly walkers and Young tableaux. II. With a wall, J. Phys. A 33 (2000), no. 48, 8835-8866. MR1801472 (2001m:82041) 
[26] _ Vicious walkers, friendly walkers, and Young tableaux. III. Between two walls, J. Statist. Phys. 110 (2003), no. 3-6, 1069-1086. MR1964695 (2004e:82027)

[27] M. Katori and H. Tanemura, Scaling limit of vicious walks and two-matrix model, Phys. Rev. E 66 (2002), no. 1, 011105 [12 pages].

[28] M. Katori, H. Tanemura, T. Nagao, and N. Komatsuda, Vicious walks with a wall, noncolliding meanders, and chiral and Bogoliubov-de Gennes random matrices, Phys. Rev. E 68 (2003), no. 2, 021112 [16 pages].

[29] M. Katori and H. Tanemura, Nonintersecting paths, noncolliding diffusion processes and representation theory, Combinatorial Methods in Representation Theory and their Applications, RIMS Kokyuroku, 1438 (2005), 83-102.

[30] P. Bak, C. Tang, and K. Wiesenfeld, Self-organized criticality, Phys. Rev. A 38 (1998), no. 1, 364-374. MR949160 (89g:58126)

[31] D. Huse and M. Fisher, Commensurate melting, domain walls, and dislocations, Phys. Rev. B (3) 29 (1984), no. 1, 239-270. MR0729979 (85e:82025)

[32] J. W. Essam and A. J. Guttmann, Vicious walkers and directed polymer networks in general dimensions, Phys. Rev. E (3) 52 (1995), no. 6, 5849-5862. MR1384738 (97b:82049)

[33] S. Yu. Grigor'ev and V. B. Priezzhev, Random walks of annihilating particles on a ring, Teoret. Mat. Fiz. 146 (2006), no. 3, 488-498; English transl., Theoret. and Math. Phys. 146 (2006), no. 3, 411-420. MR.2254671 (2007d:82036)

[34] J. W. van de Leur and A. Yu. Orlov, Random turn walk on a half line with creation of particles at the origin, arXiv:0801.0066

[35] N. M. Bogolyubov, The XXO Heisenberg chain and random walks, Zap. Nauchn. Sem. S.-Peterburg. Otdel. Mat. Inst. Steklov. (POMI) 325 (2005), 13-27; English transl., J. Math. Sci. (N. Y.) 138 (2006), no. 3, 5636-5643. MR2160317 (2006e:82008)

[36] __ Integrable models for vicious and friendly walkers, Zap. Nauchn. Sem. S.-Peterburg. Otdel. Mat. Inst. Steklov. (POMI) 335 (2006), 59-74; English transl., J. Math. Sci. (N. Y.) 143 (2007), no. 1, 2729-2737. MR2269751 (2007i:82025)

[37] N. M. Bogoliubov and C. Malyshev, A path-integration approach to the correlators of XY Heisenberg magnet and random walks, Path Integrals, World Sci. Publ., Singapore, 2008, pp. 508-513; arXiv: 0810.4816. MR2503741(2010d:82017)

[38] Correlation function of the XX Heisenberg magnet, and random walks of vicious walkers, Teoret. Mat. Fiz. 159 (2009), no. 2, 179-193; English transl. in Theoret. and Math. Phys. 159 (2009). MR2567335 (2010j:82017)

[39] E. Lieb, T. Schultz, and D. Mattis, Two soluble models of an antiferromagnetic chain, Ann. Physics 16 (1961), no. 3, 407-466. MR0136392 (24:B2430)

[40] Th. Niemeijer, Some exact calculations on a chain of spin $\frac{1}{2}$. I, II, Physica 36 (1967), no. 3, 377-419; 39 (1968), no. 3, 313-326.

[41] F. Colomo, A. G. Izergin, V. E. Korepin, and V. Tognetti, Correlators in the Heisenberg XXO chain as Fredholm determinants, Phys. Lett. A 169 (1992), no. 4, 243-247. MR1182521 (93f:82020)

F. Colomo, A. G. Izergin, V. E. Korepin, and V. Tognetti, Temperature correlation functions in the XXO Heisenberg chain. I, Teoret. Mat. Fiz. 94 (1993), no. 1, 19-51; English transl., Theoret. and Math. Phys. 94 (1993), no. 1, 11-38. MR1226428(95b:82003)

[42] A. G. Izergin, N. A. Kitanin, and N. A. Slavnov, On correlation functions of the XY model, Zap. Nauchn. Sem. S.-Peterburg. Otdel. Mat. Inst. Steklov. (POMI) 224 (1995), 178-191; English transl., J. Math. Sci. (N.Y.) 88 (1998), no. 2, 224-232. MR.1364849 (97k:82011)

[43] A. G. Izergin, V. S. Kapitonov, and N. A. Kitanin, Simultaneous temperature correlators of the one-dimensional Heisenberg XY chain, Zap. Nauchn. Sem. S.-Peterburg. Otdel. Mat. Inst. Steklov. (POMI) 245 (1997), 173-206; English transl., J. Math. Sci. (N.Y.) 100 (2000), no. 2, 2120-2140. MR 1627784 (99f:82011)

[44] V. S. Kapitonov and A. G. Pron'ko, Time-dependent correlators of local spins in the onedimensional Heisenberg XY-chain, Zap. Nauchn. Sem. S.-Peterburg. Otdel. Mat. Inst. Steklov. (POMI) 269 (2000), 219-261; English transl., J. Math. Sci. (N.Y.) 115 (2003), no. 1, 2009-2032. MR $1805863(2002 \mathrm{a}: 82026)$

[45] K. Malyshev, Functional integration with an "automorphic" boundary condition, and correlators of the third spin components in the XX Heisenberg model, Teoret. Mat. Fiz. 136 (2003), no. 2, 285-298; English transl., Theoret. and Math. Phys. 136 (2003), no. 2, 1143-1154. MR2025377 (2004k:82020) 
[46] _ Functional integration with "automorphic" boundary conditions and correlators of Z-components of spins in the $X Y$ and $X X$ Heisenberg chains, New Developments in Mathematical Physics Research, Nova Sci. Publ., Hauppauge, 2004, pp. 85-116; arXiv:math-ph/0405009 MR2076275 (2005e:82011)

[47] S. Sachdev, Quantum phase transitions, Cambridge Univ. Press, Cambridge, 1999. MR1785382 (2002h:82035)

[48] F. Colomo, A. G. Izergin, and V. Tognetti, Correlation functions in the XXO Heisenberg chain and their relations with spectral shapes, J. Phys. A 30 (1997), no. 2, 361-370. MR 1442868 (98f:82017)

[49] V. Korepin and J. Terilla, Thermodynamic interpretation of the quantum error correcting criterion, Quantum Inf. Process. 1 (2002), no. 4, 225-242. MR.1964347(2005a:94039)

[50] B.-Q. Jin and V. E. Korepin, Quantum spin chain, Toeplitz determinants and Fisher-Hartwig conjecture, J. Statist. Phys. 116 (2004), no. 1-4, 79-95. MR2083138 (2005e:82015)

[51] F. C. Alcaraz and R. Z. Bariev, An exactly solvable constrained XXZ chain, Statistical Physics on the Eve of the 21st Century, Ser. Adv. Statist. Mech., vol. 14, World Sci. Publ., Singapore, 1999; arXiv: cond-mat/9904042 MR1704014 (2000c:82026)

[52] N. I. Abarenkova and A. G. Pron'ko, The temperature correlator in the absolutely anisotropic Heisenberg XXZ-magnet, Teoret. Mat. Fiz. 131 (2002), no. 2, 288-303; English transl., Theoret. and Math. Phys. 131 (2002), no. 2, 690-703. MR1932255 (2003g:82013)

[53] A. J. A. James, W. D. Goetze, and F. H. L. Essler, Finite-temperature dynamical structure factor of the Heisenberg-Ising chain, Phys. Rev. B 79 (2009), no. 21, 214408 [20 pages].

[54] P. Lu, G. Muller, and M. Karbach, Quasiparticles in the XXZ model, arXiv:0909.2728

[55] I. G. Macdonald, Symmetric functions and Hall polynomials, Oxford Univ. Press, New York, 1995. MR 1354144 (96h:05207)

[56] F. R. Gantmacher, Theory of matrices, Nauka, Moscow, 1988; English transl. of 1st ed., Chelsea Publ. Co., New York, 1959. MR0986246 (90a:15001) MR0107649(21:6372c)

[57] D. P. Zhelobenko and A. I. Shtern, Representations of Lie groups, Nauka, Moscow, 1983; English transl., Adv. Stud. Contemp. Math., vol. 7, Gordon and Breach Sci. Publ., New York, 1990. MR0709598 (85g:22001) MR1104272 (91m:22001)

[58] M. L. Mehta, Random matrices, Acad. Press, Inc., Boston, MA, 1991. MR1083764 (92f:82002)

[59] A. Okounkov, Infinite wedge and random partitions, Selecta Math. (N. S.) 7 (2001), no. 1, 57-81. MR.1856553 (2002f:60019)

[60] G. Kuperberg, Another proof of the alternative-sign matrix conjecture, Int. Math. Res. Notices 1996, no. 3, 139-150. MR1383754 (97c:05009)

St. Petersburg Branch, Steklov Mathematical Institute, Russian Academy of Sciences, 27 Fontanka, St. Petersburg 191023, Russia

E-mail address: bogoliub@pdmi.ras.ru

St. Petersburg Branch, Steklov Mathematical Institute, Russian Academy of Sciences, 27 Fontanka, St. Petersburg 191023, Russia

E-mail address: malyshev@pdmi.ras.ru

Received 19/FEB/2010

Translated by THE AUTHORS 